\title{
Nachhaltiges Wachstum: Braucht Deutschland ein Investitionsprogramm?
}

Der Wirtschaftseinbruch bedingt durch die Corona-Pandemie trifft in Deutschland auf einen Strukturwandel, der speziell durch die notwendige Digitalisierung und Dekarbonisierung sowie den demografischen Wandel getrieben ist. Einerseits wird kurzfristig durch das CoronaKonjunkturpaket und den EU-Aufbaufonds auf die Krise reagiert. Andererseits werden eine seit längerem existierende Investitionslücke sowie Hemmnisse hinsichtlich der Abrufung vorhandener Mittel bemängelt. Die Autor:innen diskutieren, welche fiskalischen Spielräume vorhanden sind, sowie auf welche Weise und in welche Bereiche öffentliche Investitionen in Deutschland getätigt werden müssten, um nachhaltiges Wirtschaftswachstum zu ermöglichen.

\section{Unzureichende Investitionsoffensive}

Michael Hüther, Institut der deutschen Wirtschaft.

Markos Jung, Institut der deutschen Wirtschaft.

Investitionen für nachhaltiges Wachstum in Deutschland: Status quo und Perspektiven

Veronika Grimm, Friedrich-Alexander-Universität Erlangen-Nürnberg; Sachverständigenrat zur Begutachtung der gesamtwirtschaftlichen Entwicklung.

Lukas Nöh, Sachverständigenrat zur Begutachtung der gesamtwirtschaftlichen Entwicklung.

Milena Schwarz, Sachverständigenrat zur Begutachtung der gesamtwirtschaftlichen Entwicklung.

\section{Ein Investitionsprogramm zur Krisenbewältigung}

Marius Clemens, DIW Berlin.

Marcel Fratzscher, Humboldt-Universität zu Berlin; DIW Berlin.

Claus Michelsen, DIW Berlin.

Öffentliche Investitionen im Konjunkturprogramm als Einstieg in die sozial-ökologische Transformation

Sebastian Dullien, Hochschule für Technik und Wirtschaft Berlin; Institut für Makroökonomie und Konjunkturforschung der Hans-Böckler-Stiftung (IMK) Düsseldorf.

Katja Rietzler, IMK Düsseldorf.

Silke Tober, IMK Düsseldorf.

\section{Deutschland braucht ein Investitionskompetenzprogramm}

Martin Hellwig, Max-Planck-Institut zur Erforschung von Gemeinschaftsgütern Bonn. 